\title{
Serum anti-collagen type IV IgM antibodies and development of diabetic nephropathy in diabetics with essential hypertension
}

\author{
ASPARUH NIKOLOV ${ }^{1}$ IVAN TSINLIKOV ${ }^{1}$, IVANKA TSINLIKOVA ${ }^{1}$, GEORGE NICOLOFF ${ }^{2}$, \\ ALEXANDER BLAZHEV ${ }^{2}$, ANTOAN GAREV $V^{l}$ \\ ${ }^{1}$ Department of Propedeutics of Internal Diseases, Medical University, Pleven, Bulgaria \\ ${ }^{2}$ Division of Biology, Medical University, Pleven, Bulgaria
}

\begin{abstract}
Introduction and aims: Arterial hypertension and diabetic vascular complications are connected with an elevated degradation of elastic tissue. This process leads to an increased production of antibodies to collagen type IV (ACIV Abs). In the present investigation we studied whether the serum levels of antibodies (IgG, IgM and IgA) to collagen are related with microvascular complications.

Material and methods: Serum levels of antibodies to collagen type IV (ACIV) IgG, IgM and IgA were measured using an ELISA method in 93 patients with type 2 diabetes mellitus and arterial hypertension (AH) (mean age $61.4 \pm 11.3$ years, diabetes duration $9.88 \pm 3.12$ years; hypertension duration $9.28 \pm 4.98$ ). These values were compared to serum antibodies to CIV in 42 age and sex matched controls.

Results: ACIV IgM antibodies levels in patients with AH and T2DM were statisticaly significantly higher than controls $0.178(0.145 \div 0.220)$ vs. $0.142(0.118 \div 0.173)(K W=6.31 ; p=0.01)$. Group 1 (patients with microvascular complications) showed significantly higher levels of ACIV IgM than controls $0.180(0.136 \div 0.223)$ vs. $0.142(0.118 \div 0.173)(K W=5.03 ; p=0.02)$. Patients from Group 2 showed statistically significantly higher levels of ACIV IgM than controls $0.176(0.151 \div 0.202)$ vs. 0.142 $(0.118 \div 0.173)(K W=6.15 ; p=0.01)$. ACIV IgM antibodies showed correlation with microalbuminuria $(r=0.21) ;(p=0.04), B M I(r=0.19) ;(p=0.04)$, creatinine clearance $(r=-0.36) ;(p=0.01)$ and GFR $(r=-0.34) ;(p=0.02)$.

Conclusions: Our study showed an association between elevation of serum levels of ACIV IgM and development of diabetic nephropathy. We suggest that levels of ACIV IgM can be useful method for identfying a high risk for development of diabetic nephropathy.
\end{abstract}

Key words: type 2 diabetes, essential hypertension, anti-collagen type IV IgM antibodies.

(Cent Eur J Immunol 2016; 41 (1): 86-92)

\section{Introduction}

Patients with type 2 diabetes mellitus are at high risk of development of vascular disease. This risk is increased with existence of arterial hypertension. In diabetic patients morbidity and mortality are mainly related to the presence of late complications, namely macro- and microangiopathy. Arterial hypertension is connected with decreased, while type 2 diabetes mellitus is connected with an elevated degradation of connective tissue proteins Laviades et al. [1]. Because it is very important to find characteristics of pathological activation of collagen type IV turnover we studied diabetic patients with arterial hypertension who demonstrated vascular complications. In diabetes mellitus, thickening of basement membrane in capillaries and small vessels is a well-known finding and important in the progression of diabetic microangiopathy.
Collagen type IV (CIV) is uniquely present in basement membranes and represents their predominant structural element Klatt et al. [2], Coelho et al. [3]. Metabolic alteration of CIV occurs in micro- or macrovascular basement membrane of diabetic patients. Collagen type IV constitutes the major component of basement membranes Erben et al. [4]. Measurement of serum antibodies to fragments of CIV Nikolov et al. [5]; Nicoloff et al. [6] is now possible and enables changes to be detected. Investigators Kanie et al. [7] measured serum anti-type IV collagen antibody $(\mathrm{IgG})$ in diabetic patients and they found that serum levels of the anti-type IV collagen antibody were significantly higher in diabetics than these in the nondiabetics. However, there was no relationship between the levels of urinary albumin and the serum levels of anti-type IV collagen antibody.

Correspondence: Asparuh Nikolov, Department of Propedeutics of Internal Diseases, Medical University, Pleven,

e-mail: a_nicoloff@yahoo.com

Submitted: 16.07.2015; Accepted: 6.11.2015 
Authors Morillas et al. [8] study circulating biomarkers of collagen metabolism in arterial hypertension: relevance of target organ damage. Their study is based on the increasing experimental evidence that alterations in the extracellular matrix are implicated in hypertension and its chronic complications. Selected markers of extracellular matrix metabolism were investigated as potential biomarkers for hypertensive remodelling and correlated with the severity and extent of target organ damage (TOD) in patients with essential hypertension. Authors studied 159 consecutive patients being treated for essential hypertension. An exhaustive evaluation of the heart, kidney and blood vessel damage was performed, and plasma levels of plasma procollagen type I (PICP), matrix metalloproteinase-1 (MMP-1) and its inhibitor (tissue inhibitor metalloproteinase-1 - TIMP-1) were determined. Patients were categorized into four groups: no TOD (33 patients), level 1 TOD (52 patients), level 2 TOD (44 patients) and level 3 TOD (30 patients). The serum levels of MMP-1 and TIMP-1 were higher in patients with TOD than in hypertensive patients without TOD. Increasing levels of these molecules were progressively associated with an increase in the number of organs damaged, with highest levels of markers in patients with level 3 TOD (heart, kidney and blood vessels). There were no differences in PICP levels between groups. We found a slight but significant correlation between TIMP-1 and all hypertensive organ damage. Logistic regression analysis showed that age, smoking, diabetes mellitus, abdominal perimeter, MMP-1 and TIMP-1 were independently related to the level of TOD. Authors conclude that circulating concentration of MMP-1 and TIMP-1 is associated with an extended hypertensive disease, with more TOD. TIMP-1 may have a role as a biomarker of total remodelling burden in hypertensive patients.

In diabetic patients morbidity and mortality are mainly related to the presence of late complications, namely macro- and microangiopathy. Microvascular disease was defined as the pesence of eighter retinopathy or urinary albumin excretion rate of more than $20 \mu \mathrm{g} / \mathrm{min}$ The Framingham study 1979 [9]. Thickening of basement membrane in capillaries and small vessels is a well-known finding and important in the progression of diabetic microangiopathy. Collagen type IV represents predominant structural element of basement membranes Kühn Rosenbloom et al. $[10,11]$. It is known, that CIV macromolecule is not fully degradated by collagenases in vivo. These CIV may become a stimulus for immunocompetent cells and result in the production of anti-CIV antibodies.

Diabetic nephropathy is leading cause of end stage of renal disease (ESRD) in the USA. Most forms of ESRD can be prevented. Diabetic nephropathy affects $20-30 \%$ of patients with type 2 diabetes mellitus. The average 5-year survival for patients expected to develop ESRD because of diabetes mellitus, is less than $20 \%$. This increased risk of mortality may be due to the renal disease, which can be considered to be the cause of about $50 \%$ of mortality. Diabetic nephropathy is one of the most serious complications of diabetes. It is defined as persistent albuminuria over $300 \mathrm{mg} / 24 \mathrm{~h}$ or $200 \mu \mathrm{g} / \mathrm{min}$ at a reduced glomerular filtration rate and elevated blood pressure - ADA, 2001 [12].

There is data for detection of serum CIV fragments in animals and humans with arterial hypertension. These fragments can be a stimulus for immunocompetent cells for pathological production of anti-CIV antibodies. Researchers Susumu et al. [13] have measured the serum anti-CIV $\mathrm{IgG}$ in diabetics and have found that these levels were significantly more in diabetics than in healthy controls. However, no correlation between level of albuminuria and serum levels of anti CIV IgG was found. We investigated anti-CIV antibodies in children with essential hypertension. The patients showed significantly higher levels of anti-CIV IgM, while the levels of anti-CIV IgG were lower than the controls Nicoloff et al. [14].

In this study, we tried to find out whether serum antibodies (IgM, IgG and IgA) to CIV epitopes associate with the development of microangiopathy in diabetic patients with arterilal hypertension. This study established the normal serum levels of anti-CIV in healthy subjects and compare the results with those of patients. The observed changes correlate with microvascular complications.

\section{Material and methods}

The sera of 42 clinically healthy subjects (mean age $58.9 \pm 7.56$ ) were used as controls. The selected controls were individuals with no family history of arterial hypertension, diabetes mellitus and atherosclerosis, who did not suffer from any inflammatory processes, collagenoses, and emphysema and had no history of hepatitis. Their routine clinical examinations, lipid profile and serum proteins showed no changes, ECG was normal.

Apart from the clinically healthy individuals, 93 patients with type 2 diabetes mellitus and arterial hypertension were also examined. Their mean age was $61.4 \pm 11.3$ years; the duration of diabetes was $9.88 \pm 3.12$ years, and of arterial hypertension $-9.28 \pm 4.98$ years. They were all from the region of the Medical University in Pleven. The diabetic patients were divided into two groups according to the presence - Group 1 $(n=67)$ or absence - Group $2(n=26)$ of microangiopathy (Tables 1, 2, Fig. 1). An ethical approval was obtained from the Ethics Committee, and the patients signed informed consent forms for their participation in the research.

\section{Procedures}

\section{ELISA (enzyme-linked immunosorbent assay)}

- Serum antibodies (IgG, IgM and IgA) to CIV were measured by an enzyme-linked immunosorbent assay (ELISA). In brief, each well of the microtiter plate was sensi- 
Asparuh Nikolov et al.

Table 1. Clinical data of patients with type 2 diabetes mellitus and arterial hypertension

\begin{tabular}{|c|c|c|c|}
\hline Clinical data & Group 1 & Group 2 & Controls \\
\hline Age & $62.5 \pm 12.58$ & $60.4 \pm 8.4$ & $58.9 \pm 7.56$ \\
\hline Gender (M/F) & $26 / 41$ & $11 / 15$ & $20 / 22$ \\
\hline Mean diabetes duration & $9.30 \pm 5.36$ & $9.16 \pm 7.59$ & N/A \\
\hline Mean hypertension duration & $9.50 \pm 7.63$ & $8.68 \pm 7.26$ & N/A \\
\hline $\mathrm{HbA}_{1 \mathrm{c}}$ (glycated haemoglobin) & $7.63 \pm 2.03$ & $7.27 \pm 1.63$ & N/A \\
\hline Systolic blood pressure ( $\mathrm{mmHg}$ ) & $142.83 \pm 18.05$ & $140.58 \pm 20.51$ & $114.29 \pm 15.74$ \\
\hline Diastolic blood pressure $(\mathrm{mmHg})$ & $82.23 \pm 11.52$ & $81.35 \pm 11.96$ & $72.5 \pm 10.4$ \\
\hline BMI (body mass index) & $29.62 \pm 4.99$ & $28.42 \pm 3.96$ & $22.61 \pm 2.27$ \\
\hline Total cholesterol (mmol/l) & $5.26 \pm 1.40$ & $5.18 \pm 0.93$ & $3.99 \pm 0.65$ \\
\hline HDL (mmol/l) & $0.88 \pm 0.30$ & $0.93 \pm 0.30$ & $0.96 \pm 0.20$ \\
\hline $\mathrm{LDL}(\mathrm{mmol} / \mathrm{l})$ & $3.18 \pm 1.19$ & $3.16 \pm 1.09$ & $2.43 \pm 0.64$ \\
\hline Triglycerides (mmol/l) & $2.91 \pm 1.68$ & $2.53 \pm 1.49$ & $1.31 \pm 0.61$ \\
\hline Insulin dose $(\mathrm{U} / \mathrm{kg} / 24 \mathrm{~h})$ & $2.57 \pm 0.52$ & $2.03 \pm 0.93$ & N/A \\
\hline Serum creatinine (umol/l) & $102.98 \pm 27.12$ & $97.33 \pm 19.24$ & \\
\hline Creatinine clearance (Cockroft and Gault Formula) (ml/min) & $66.76 \pm 26.42$ & $67.18 \pm 21.51$ & \\
\hline Glomerular filtration rate $\left(\mathrm{ml} / \mathrm{min} / 1.73 \mathrm{~m}^{2}\right)$ & $62.87 \pm 19.66$ & $63.42 \pm 14.38$ & \\
\hline Anti-CIV IgM (ng/ml) & $\begin{array}{c}0.180 \\
(0.136 \div 0.223)\end{array}$ & $\begin{array}{c}0.176 \\
(0.151 \div 0.202)\end{array}$ & $\begin{array}{c}0.142 \\
(0.118 \div 0.173)\end{array}$ \\
\hline $\operatorname{MAU}(\mu \mathrm{g} / \mathrm{min})$ & $* 78.94 \pm 52.87$ & $8.53 \pm 4.69$ & N/A \\
\hline MAU (microalbuminuria) & $(n=43)$ & - & \\
\hline Retinopathy & $(n=20)$ & - & \\
\hline Neuropathy & $(n=4)$ & - & \\
\hline Smokers & $37 / 67$ & $15 / 26$ & $16 / 42$ \\
\hline Percentage smokers $(\mathrm{M} / \mathrm{F})$ & $55 \% / 45 \%$ & $58 \% / 42 \%$ & $47 \% / 53 \%$ \\
\hline Number & 67 & 26 & 42 \\
\hline Percentage (M/F) & $39 \% / 61 \%$ & $42 \% / 58 \%$ & $45 \% / 55 \%$ \\
\hline
\end{tabular}

Group 1 - patients with microvascular complications ( $n=67)$; Group 2 - patients without microvascular complications $(n=26)$; Controls $(n=42)$;

Values are mean $\pm S D$

Table 2. Levels of serum anti-CIV IgM in patients with type 2 diabetes mellitus and arterial hypertension

\begin{tabular}{lcccc}
\hline \multirow{2}{*}{ Groups } & \multicolumn{2}{c}{ Anti-CIV IgM $(\mathbf{n g} / \mathbf{m l})$} & \multicolumn{2}{c}{ Comparison to other groups } \\
\cline { 2 - 5 } & $\mathbf{M} \div(\mathbf{Q 1}-\mathbf{Q 3})$ & Group 1 & Group 2 & All diabetics \\
\hline All diabetics & 0.178 & NS & NS & NS \\
\hline Group 1 & $0.145 \div 0.220)$ & & NS & NS \\
\hline Group 2 & 0.180 & - & - & $p=0.01$ \\
\hline Controls & $0.136 \div 0.223)$ & NS & & $p=0.01$ \\
\hline
\end{tabular}

Values are median together with first and third quartile Q1 and Q3; (twenty-fifth and seventy-fifth percentile P25 and 75P) 
tized with $100 \mu \mathrm{l}$ of $10 \mu \mathrm{g} / \mathrm{ml}$ of human CIV (SIGMA, USA) at room temperature for $3 \mathrm{~h}$, followed by an overnight incubation at $4^{\circ} \mathrm{C}$.

- The plate was washed with phosphate-buffered saline (PBS) containing $0.05 \%$ Tween 20 and $1 \%$ bovine serum albumin (BSA, SIGMA, USA).

- Then, $100 \mu \mathrm{l}$ serum sample (diluted $1: 10$ ), was placed in each well of a microtiter plate, and incubated for $1 \mathrm{~h}$ at $37^{\circ} \mathrm{C}$.

- After washing three times, $100 \mu \mathrm{l}$ of immunoconjugates (anti-human immunoglobulin peroxidase conjugates (SIGMA, USA) to heavy chain of $\operatorname{IgG}, \operatorname{IgM}$ and $\operatorname{IgA}$ ) were added to each well for $1 \mathrm{~h}$ at $37^{\circ} \mathrm{C}$. All immunoconjugates were diluted $1: 10,000$ with PBS containing $1 \%$ BSA and $0.05 \%$ Tween 20.

- The plate was incubated for $1 \mathrm{~h}$ at $37^{\circ} \mathrm{C}$. o-Phenylenediamine $(0.4 \mathrm{mg} / \mathrm{ml})$ was added to citrate buffer, and $100 \mu \mathrm{l}$ of this solution was added to each well and allowed to react for $30 \mathrm{~min}$.

- The reaction was stopped by adding $50 \mu \mathrm{l} 4 \mathrm{M} \mathrm{H}_{2} \mathrm{SO}_{4}$ to each well and the optical density was measured with a Microelisa Reader 210 (Organon Teknika, Belgium) at a wavelength of $492 \mathrm{~nm}$.

\section{Other tests performed}

1. Ophthalmoscopy through dilated pupils was carried out in all diabetic patients by the same ophthalmologist.

2. Glycated haemoglobin was measured using high-pressure liquid chromatography (normal range 4-6\%).

3. Serum total cholesterol and triglyceride concentrations were measured by enzyme assay (Boehringer Mannheim, Mannheim, Germany).

4. Arterial blood pressure was measured using a standard mercury sphygmomanometer, to the nearest $2 \mathrm{~mm} \mathrm{Hg}$, in the dominant arm after at least $10 \mathrm{~min}$ rest in the supine position.

5. AER (albumin excretion rate) was determined by nephelometry using a commercial kit containing specific antibody (Behringwerke AG, Marburg, Germany).

\section{Statistical analysis}

The research data was processed with the computer programs EXCEL (Microsoft Corporation, Redmond, WA) and STATGRAPHICS plus (Manugistics, Rockville, MD) for WINDOWS. All results were described in tables, graphs, numerical values (mean $\pm \mathrm{SD}$, share indicators and correlations). For assessment and conclusions in the case of normal distribution the Student $t$-test, Fisher's $F$-test (ANOVA) and post-hoc tests (LSD, Tukey HSD, Scheffe, Bonferroni, Newman-Keuls, and Duncan) were used, and for distribution, different from the normal - the K-W (Kruskal-Wallis)-test. The level of significance was determined as $p<0.05$. In cases with different from normal distribution, median was used $(\mathrm{M})$, together with first and third

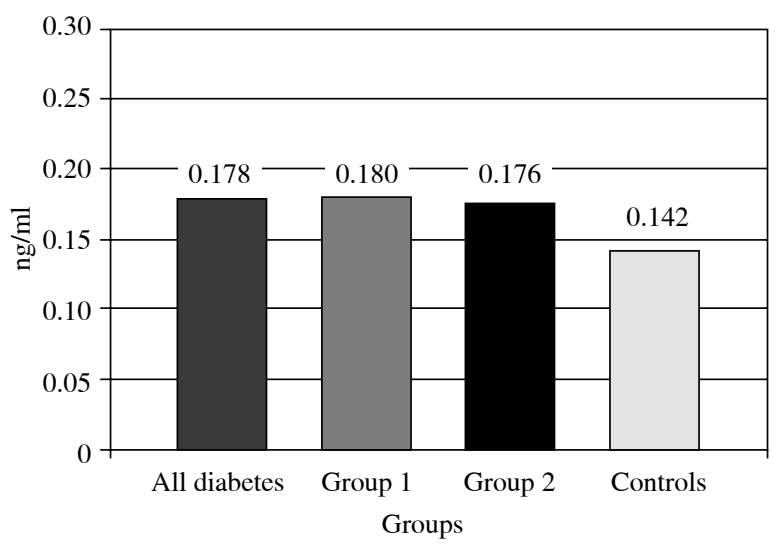

Values are median together with first and third quartile Q1 and Q3; (twenty-fifth and seventy-fifth percentile $P 25$ and $75 P$ )

Fig. 1. Serum Anti-CIV IgM levels in patients with type 2 diabetes mellitus and arterial hypertension

quartile Q1 and Q3; (twenty-fifth and seventy-fifth percentile P25 and 75P).

\section{Results}

Anti-collagen type IV (ACIV) IgM antibodies levels in patients with arterial hypertension $(\mathrm{AH})$ and type 2 diabetes mellitus (T2DM) were statisticaly significantly higher than controls $0.178(0.145 \div 0.220)$ vs. $0.142(0.118 \div 0.173)$ $(\mathrm{KW}=6.31 ; p=0.01)$. Group 1 (patients with microvascular complications) showed significantly higher levels of ACIV IgM than controls $0.180(0.136 \div 0.223)$ vs. $0.142(0.118 \div 0.173)(\mathrm{KW}=5.03 ; p=0.02)$. Patients from Group 2 showed statistically significantly higher levels of ACIV IgM than controls $0.176(0.151 \div 0.202)$ vs. 0.142 $(0.118 \div 0.173)(\mathrm{KW}=6.15 ; p=0.01)$.

ACIV IgM antibodies showed correlation with MAU (microalbuminuria) $(r=0.21)$; $(p=0.04)$, BMI $(r=0.19)$; $(p=0.04)$, creatinine clearance $(r=-0.36) ;(p=0.01)$ and GFR (glomerular filtration rate) $(r=-0.34) ;(p=0.02)$. Serum ACIV IgG levels were higher in patients than in controls, while serum ACIV IgA levels were lower than these in controls, but the differences are not statistically significant.

\section{Discussion}

Arterial hypertension and diabetes mellitus are connected with an elevated degradation of elastic tissue, loss of elasticity, increasing rigidity of arterial wall, and abnormal increase in the collagen/elastin ratio. As a result soluble collagen type IV (CIV) derived peptides are released in the circulated blood, which are a pathological stimulus for an increased production of anti-collagen type IV (anti-CIV Abs) antibodies Nicoloff et al. [15]. In a recent investigation all diabetics showed statistically significantly higher levels of anti-CIV IgM than controls. Patients with mi- 
crovascular complications showed statistically significant higher levels of anti-CIV IgM than healthy controls. Patients from Group 2 also showed higher levels of anti-CIV IgM than healthy controls. Anti-CIV IgM correlate with microalbuminuria, BMI.

The prevalence of microalbuminuria in patients with T2DM is a sensetive predictor of future development of diabetic nephropathy. Microalbuminuria not only implies the presence of renal involvment in diabetes, but is also associated with generalized vascular damage Nikolov [16]. Increased synthesis or increased degradation could cause the elevated serum levels of CIV which are strong pathological stimulus for production of specific antibodies found in our study, mainly in patients with microalbuminuria. The circulating CIV molecules may eighter be released from tissue during synthesis, provided that the vessel wall allows such leakage, or be products of degradation of pre-existing basement membranes or newly synthesized molecules. The published data describes an increased production of CIV and protease inhibitors, but a decreased collagenolytic activity, which suggests that the first possibility, may be valid. In the present study our aim was to determine if elevation of antibodies against CIV.

Collagens are known to be immunogenic in animals, and antibodies to various collagens are present in human serum in several diseases, particularly those of autoimmune origin Patel et al. [17], González et al. [18]. The detection of serum CIV in animals or humans with arterial hypertension Yano et al. [19] shows that CIV macromolecule is not fully degraded by collagenases in vivo. These CIV fragments may become an increased stimulus for immunocompetent cells for pathological production of anti-CIV antibodies. Changes in the levels and pattern of antibodies to CIV may occur with time.

Serum type IV collagen in diabetic patients at risk for nephropathy is examined by Cohen et al. [20]. Whereas increased urinary excretion of type IV collagen, which is believed to reflect renal overproduction of this extracellular matrix protein in early diabetic nephropathy, has been confirmed in several studies, examination of serum concentrations of this analyte has yielded conflicting results. Authors sought to clarify the relationship between early renal dysfunction in diabetes and circulating type IV collagen concentrations. Authors measured serum (human) collagen IV concentrations by immunoassay in 109 patients with type 1 or type 2 diabetes and various amounts of albuminuria extending from the normo- to the macroalbuminuric range, and they examined its relationship to albumin excretion and to serum creatinine levels. Serum collagen IV concentrations (mean 6 SEM) were not significantly different in normoalbuminuric $(219 \pm 10 \mathrm{ng} / \mathrm{ml})$, microalbuminuric (209 $\pm 6 \mathrm{ng} / \mathrm{ml})$, or macroalbuminuric (206 $\pm 7 \mathrm{ng} / \mathrm{ml}$ ) diabetic subjects or in nondiabetic normal volunteers $(206 \pm 10 \mathrm{ng} / \mathrm{ml})$. Collagen IV concentrations showed no significant correlation $(p>0.25)$ with albumin excretion $(r=-0.001), \mathrm{HbA}_{1 \mathrm{c}}(r=0.030)$, or serum creatinine $(r=-0.161)$ and were unrelated to urinary excretion of collagen IV in the subset of subjects in whom these data were available. The results of this cross-sectional analysis discount the utility of measurement of the serum concentration of collagen IV as an indicator of early renal dysfunction in diabetes. Increased urine excretion of collagen IV without a significant change in the serum concentration is consistent with a renal origin of this analyte in early diabetic nephropathy.

Investigators Araki et al. [21] study association between urinary type IV collagen level and deterioration of renal function in type 2 diabetic patients without overt proteinuria. Cross-sectional studies have reported increased levels of urinary type IV collagen in diabetic patients with progression of diabetic nephropathy. The aim of this study was to determine the role of urinary type IV collagen in predicting development and progression of early diabetic nephropathy and deterioration of renal function in a longitudinal study. Japanese patients with type 2 diabetes ( $n=254,185$ with normoalbuminuria and 69 with microalbuminuria) were enrolled in an observational follow-up study. The associations of urinary type IV collagen with progression of nephropathy and annual decline in estimated glomerular filtration rate (eGFR) were evaluated. At baseline, urinary type IV collagen levels were higher in patients with microalbuminuria than in those with normoalbuminuria and correlated with urinary $\beta(2)$ microglobulin $(\beta=0.57, p<0.001)$, diastolic blood pressure $(\beta=0.15$, $p<0.01)$, eGFR $(\beta=0.15, p<0.01)$, and urinary albumin excretion rate $(\beta=0.13, p=0.01)$ as determined by multivariate regression analysis. In the follow-up study (median duration 8 years), urinary type IV collagen level at baseline was not associated with progression to a higher stage of diabetic nephropathy. However, the level of urinary type IV collagen inversely correlated with the annual decline in eGFR $(\gamma=-0.34, p<0.001)$. Multivariate regression analysis identified urinary type IV collagen, eGFR at baseline, and hypertension as factors associated with the annual decline in eGFR. The results indicate that high urinary excretion of type IV collagen is associated with deterioration of renal function in type 2 diabetic patients without overt proteinuria.

The rise in serum levels of anti-CIV IgM indicates abnormality of collagen metabolism. Increased synthesis and/ or increased degradation of CIV would cause rising serum CIV fragments that are strong pathological stimulus for the production of specific antibodies found in our study, especially in patients with diabetic nephropathy. In the present study, the levels of IgM anti-CIV were statistically significantly higher in patients with microalbuminuria. It is possible these patients to be in the early stages of the development of macrovascular disease. Moreover, in our study we found correlation of of anti-CIV IgM with microalbuminuria and GFR (correlation with GFR os reverse, 
which is very important because with anti-CIV IgM increase GFR would decrase, confirming renal dysfunction). That is why we suggest that serum anti-CIV IgM may be a useful marker for detection of early diabetic nephropathy.

The first immunoglobulin synthesized during the early phase of a pathologically activated immune response is $\operatorname{IgM}$. The immune system then swithces on to production of $\mathrm{IgG}$. Interestingly we found significant elevation of anti-CIV IgM in secondary immune response. We did not observe the character kinetics of IgM levels. The increased anti-CIV IgM during secondary immune response can be result of:

1. Decreased CIV degradation in arterial hypertension.

2. Increased production of IgM during the secondary immune response (In some conditions like blood transfusion, primary immune response is not always characterised by production of IgM. Sometimes only IgG can be detected for example IgG antibodies in Duffy or Kiu system. Similarly to that secondary immune response is not always characterised by only production of $\operatorname{IgG}$. Sometimes IgM is only produced, for example IgM antibodies in MNS and Lewis system).

We believe that in our study it was possible to observe the 'active' phase of vascular complications, because the levels of anti-CIV IgM are increased, while in the chronic period last levels are low and only those of anti-IgG CIV are increased. Our study has shown a link between serum levels of anti-CIV IgM and the development of diabetic nephropathy. The presence of the above antibodies would lead to diverse pathological processes, including the formation of immune complexes, complement activation, and activation of the K-cell-mediated antibody-dependent cellular cytotoxicity. All of these processes may contribute to the destruction of the collagen in the arterial wall. Moreover, collagen antigens presented on the damaged areas of the vessel wall are a target for the specific $\mathrm{T}$ and B lymphocytes, which could enhance the immune response Peterszegi et al. [22].

Diabetic nephropathy (DN) is believed to be a major microvascular complication of diabetes Hu et al. [23]. The hallmark of DN includes deposition of extracellular matrix (ECM) proteins, such as, collagen, laminin and fibronectin in the mesangium and renal tubulo-interstitium of the glomerulus and basement membranes. Such an increased expression of ECM leads to glomerular and tubular basement membranes thickening and increase of mesangial matrix, ultimately resulting in glomerulosclerosis and tubulointerstitial fibrosis. Thus, the accumulation of ECM proteins plays a critical role in the development of DN. ECM synthesis and its expression in high glucose ambience in vitro and in vivo states. The understanding of such signaling pathways and the molecules that influence expression, secretion and amassing of ECM may aid in developing strategies for the amelioration of diabetic nephropathy.
Authors Iida et al. [24] studied hypertensive patients with early prediabetes $\left(\mathrm{HbA}_{1 \mathrm{c}}<5.7 \%\right.$ and fasting glucose $>110, n=18)$, those with prediabetes $\left(\mathrm{HbA}_{1 \mathrm{c}}\right.$ 5.7-6.4, $n=98)$, and those with diabetes $\left(\mathrm{HbA}_{1 \mathrm{c}}>6.5\right.$ or on diabetes medications, $n=92$ ). The participants underwent echocardiography to assess left atrial volume/body surface area (BSA) and the ratio of early mitral flow velocity to mitral annular velocity (E/e'). Urinary type IV collagen and albumin increased in parallel with the deterioration of glycemic status. In hypertensive patients with prediabetes, subjects with left ventricular diastolic diameter (LVDD) had higher levels of BNP and urinary type IV collagen than those without LVDD. In contrast, in hypertensive patients with diabetes, subjects with LVDD had higher urinary albumin and BNP than those without LVDD. Urinary type IV collagen correlated positively with BNP in hypertensive patients with prediabetes, whereas it correlated with $\mathrm{HbA}_{1 \mathrm{c}}$ in those with diabetes. Authors conclude that In hypertensive patients with prediabetes, urinary type IV collagen was associated with LV diastolic dysfunction and BNP.

Enomoto et al. in 2012 [25] studied the relationship between renal hemodynamics and urinary type IV collagen in patients with essential hypertension. Authors investigated the relationship between CIV and renal hemodynamics in 42 patients with essential hypertension. The renal resistive index (RI) is calculated from blood flow velocities measured using pulsed-wave in interlobar arteries. There was a significant correlation between CIV to creatinine ratio and age, $\mathrm{HbA}_{1 \mathrm{c}}$, and RI. A stepwise regression analysis showed that RI was independently associated with CIV/ creatinine ratio. These results indicated that urinary CIV may be a marker of renovascular stiffness due to glomerulosclerosis in patients with essential hypertension.

It is well known that diabetic nephropathy (DN) is a serious complication of diabetes associated with increased risk of mortality, and cardiovascular and renal outcomes. Diagnostic markers to detect DN at early stage are important as early intervention can slow loss of kidney function and improve patient outcomes. Urinary biomarkers may be elevated in diabetic patients even before the appearance of microalbuminuria, and can be used as useful marker for detecting nephropathy in patients with normoalbuminuria. Fiseha [26] reviewed some new and important urinary biomarkers, such as: Neutrophil gelatinase associated lipocalin (NGAL), N-acetyl-beta-glucosaminidase (NAG), Cystatin C, alpha 1-microglobulin, immunoglobulin $\mathrm{G}$ or $\mathrm{M}$, type IV collagen, nephrin, angiotensinogen and liver-type fatty acid-binding protein (L-FABP) associated with early DN in type 2 diabetic patients. Their search identified a total of 42 studies that have been published to date. Urinary levels of these biomarkers were elevated in type 2 diabetic patients compared with non-diabetic controls, including in patients who had no signs indicating nephropathy (without microalbuminuria), and showed positive correlation with albuminuria. Despite the promise of 
these new urinary biomarkers, further large, multicenter prospective studies are still needed to confirm their clinical utility as a screening tool for early type $2 \mathrm{DN}$ in every day practice.

In this study we found that the values of anti-CIV IgM in patients with vascular complications are significantly higher than in healthy controls. This means that the diabetics from Group 1 show pathologically high immune response to collagen type IV. Although the data from Group 2 reveals levels of anti-CIV IgM higher than those of the controls, these levels still remain lower than the measured in patients with vascular complications from Group 1. It is extremely important to monitor if the group of patients with high levels of anti-CIV IgM but without vascular lesions will develop impaired renal function.

In conclusion, our results suggest an association between level of anti-collagen type IV IgM antibodies and the development of vascular wall lesions. Elevation of anti-CIV IgM may indicate increased collagen turnover and development of microvascular complications. However, a larger study is necessary for clarification of these possibilities.

\section{The authors declare no conflict of interest.}

\section{References}

1. Laviades C, Varo N, Fernandez J, et al. (1998): Abnormalities of the extracellular degradation of collagen type I in essential hypertension. Circulation 98: 535-540.

2. Klatt AR, Becker AK, Neacsu CD, Paulsson M (2011): The matrilins: modulators of extracellular matrix assembly. Int J Biochem Cell Biol 43: 320-330.

3. Coelho NM, González-García C (2011) Arrangement of type IV collagen on $\mathrm{NH} 2$ and $\mathrm{COOH}$ functionalized surfaces Biotechnol Bioeng 108: 3009-3018.

4. Erben U, Neumann U, Schuppan D (2011): Hydroxyproline-containing collagen analogs trigger the release and activation of collagen-sequestered proMMP-2 by competition with prodomain-derived. Fibrogenesis Tissue Repair 4: 211-215.

5. Nikolov AG, Nicoloff G, Tsinlikov I, Tsinlikova I (2012): Anti-collagen type IV antibodies and the development of microvascular complications in diabetic patients with arterial hypertension. J IMAB 18: 144-148.

6. Nicoloff G, Deliiyski T, Nikolov A (2010): Detection of serum collagen type IV and elastin derived peptides in patients with breast cancer. Diabetologia Croatica 41: 103-111.

7. Kanie K, Narita Y, Zhao Y, Kuwabara F (2012): Collagen type IV specific tripeptides for selective adhesion of endothelial and smooth muscle cells. Biotechnol Bioeng 109: 18081816.

8. Morillas P, Quiles J, de Andrade H, et al. (2013): Circulating biomarkers of collagen metabolism in arterial hypertension: relevance of target organ damage. J Hypertens 31: 1611-1617.

9. Kannel WB, Mcgee DL (1979): Diabetes and glucose tolerance as risk factors for cardiovascular disease: The Framingham Study. Diabetes Care; 2: 120-126.
10. Kühn K (1995): Basement membrane(type IV) collagen. Matrix Biol 14: 439-450.

11. Rosenbloom J, Abrams WR, Mecham R (1993): Extracellular matrix for the elastic fiber. Faseb J 7: 165-168.

12. American Diabetes Association. Diabetes (2001) Vital Statistics. Alexandria, VA, ADA.

13. Susumu T, Uchugu T, Shoichi T (2003): Measurement of serum anti-type IV collagen antibody in diabetic patients. Annals of Gunma University School of Health Sciences 24: 87-91.

14. Nicoloff G, Baydanoff S, Petrova C, Christova P (2002): Serum antibodies tocollagen type IV and development ofdiabetic vascular complications inchildren with type 1 (insulin-depen-dent) diabetes mellitus - a longitudinalstudy. Vascular Pharmacology 38: 143-147.

15. Nicoloff G, Baydanoff S, Stanimirova N, et al. (2001): Serum collagentype IV in healthy children and childrenwith insulin-dependent diabetesmellitus. Clin Appl Immunol Invest 1: 165-168.

16. Nicoloff G, Nikolov A, Dekov D (2005): Serum AGE-elastin derived peptides among diabetic children. Vascul Pharmacol 43: 193-197.

17. Patel D, Menon R, Taite LJ (2010): Self-assembly of elastin-based peptides into the ECM: the importance of integrins and the elastin binding protein in elastic fiber assembly, Biomacromolecules, ACS Publications.

18. González GE, Rhaleb NE, Nakagawa P, et al. (2013): N-acetyl-seryl-aspartyl-lysyl-proline reduces cardiac collagen cross-linking and inflammation in angiotensin II-induced hypertensive rats. Clin Sci (Lond) 126: 85-94.

19. Yano Y, Hamanaka R, Nakamura M (2012): Smad, but not MAPK, pathway mediates the expression of type I collagen in radiation induced fibrosis. Biochem Biophys Res Commun 418: 457-463.

20. Cohen P, Shearman CW, Lautenslager GT (2001): Serum type IV collagen in diabetic patients at risk for nephropathy. Diabetes Care 24: 1324-1327.

21. Araki S, Haneda M, Koya D, et al. (2010): Association between urinary type IV collagen level and deterioration of renal function in type 2 diabetic patients without overt proteinuria. Diabetes Care 33: 1805-1810.

22. Peterszegi G, Mandet C, Texier S, et al. (1997): Lymphocytesin human atherosclerotic plaque exhibitthe elastin-laminin receptor:potentialrole in atherogenesis. Atherosclerosis 135: 103-107.

23. Hu C, Sun L, Han Y, et al. (2015): Insights into the mechanisms involved in the expression and regulation of extracellular matrix proteins in diabetic nephropathy. Curr Med Chem 22: 24-28.

24. Iida M, Yamamoto M, Ishiguro YS, et al. (2014): Urinary type IV collagen is related to left ventricular diastolic function and brain natriuretic peptide in hypertensive patients with prediabetes. J Diabetes Complications 28: 824-830.

25. Enomoto D, Okura T, Nagao T, et al. (2012): Relationship between renal hemodynamics and urinary type IV collagen in patients with essential hypertension. Clin Exp Hypertens 34: 612-616.

26. Fiseha T (2015): Urinary biomarkers for early diabetic nephropathy in type 2 diabetic patients. Biomark Res 3: 16 . 